\title{
Utilidad de amilasa y lipasa en diagnóstico y tratamiento de dolor abdominal agudo
}

\author{
MARCO FUEL HERRERA ${ }^{* *}$, GABRIELA GUALPA JÁCOME ${ }^{1,2}$, INÉS ECHEVERRÍA LLUMIPANTA \\ 'Facultad de Ciencias Químicas, Universidad Central del Ecuador, Quito-Ecuador. \\ 2Laboratorio Clínico Northospital, Av. De la Prensa y Vaca de Castro, Quito-Ecuador \\ *Correspondencia: marco_ofh_@hotmail.com
}

Recibido: 04 de abril de 2017, Aceptado: 15 de diciembre de 2017

\section{Resumen}

El objetivo del trabajo de investigación fue evaluar la utilidad de la amilasa y lipasa sérica en el diagnóstico y control de tratamiento de pacientes con dolor abdominal agudo de origen pancreático y no pancreático que acudieron al Hospital NORTHOSPITAL de Quito durante el periodo comprendido entre octubre a diciembre del 2015 y que presentaron valores elevados de amilasa y lipasa sérica. Las variables edad, sexo, antecedentes patológicos y familiares, motivo de consulta, signos y síntomas, pruebas de laboratorio e imagen, diagnóstico y tratamiento fueron obtenidas de las historias clínicas. Para el tratamiento estadístico se emplearon herramientas como: media, frecuencia, sensibilidad, especificidad, valor predictivo positivo (VPP), valor predictivo negativo (VPN), curvas $R O C$, prueba t para muestras relacionadas y ANOVA de un factor. Utilizando pruebas estadísticas de rigor se evidenció que la lipasa sérica presenta una mayor sensibilidad (90,91\%) frente a la amilasa (63,3\% de sensibilidad) para el diagnóstico de pancreatitis. Ambos parámetros bioquímicos ofrecen un 100\% de especificidad. La amilasa y lipasa sérica se elevan en el curso de una pancreatitis aguda y en otras patologías no pancreáticas, por lo tanto se precisa de otros exámenes de laboratorio y pruebas de imagen para logar un diagnóstico eficaz.

Palabras claves: Dolor abdominal agudo, pancreatitis, amilasa sérica, lipasa sérica.

\section{Usefulness of amylase and lipase in the diagnosis and treatment of acute abdominal pain}

\section{Abstract}

The objective of the research was to evaluate how useful is the amylase and serum lipase in the diagnosis and control of treatment of patients with acute abdominal pancreatic and non-pancreatic pain who attended at NORTHOSPITAL Hospital in Quito during the period October to December 2015 and had elevated serum amylase and lipase serum. The variables: age, gender, pathological and family history, reason for consultation, signs and symptoms, laboratory and imaging testing, diagnosis and treatment were obtained from medical records. Tools like: median, frequency, sensitivity, specificity, positive predictive value (PPV), negative predictive value (NPV), ROC curves, t-test for related and ANOVA samples of a factor were employed in the statistical part of this study. It was evidenced, using required statistical tests, that serum lipase has a higher sensitivity $(90.91 \%)$ compared to amylase (63.3\% sensitivity) for pancreatitis diagnosis. Both biochemical parameters offer a 100\% specificity. Serum amylase and lipase are elevated in acute pancreatitis and in other non-pancreatic pathologies; therefore other lab and imaging test are needed in order to achieve an effective diagnosis.

Key words: Acute abdominal pain, pancreatitis, serum amylas, serum lypase 


\section{Introducción}

En la actualidad el dolor abdominal agudo es el principal motivo de consulta en el ámbito hospitalario, constituyendo el $50 \%$ de las atenciones en servicio de urgencia hospitalaria [1]. Según el Instituto Nacional de Estadística y Censos del Ecuador en el 2014 la pancreatitis de etiología biliar estuvo dentro de las 5 principales causas de morbilidad en el país; por cada 100 egresos, la tasa de letalidad hospitalaria se distribuye en: 1.93 para el dolor abdominal agudo y 2.47 para la pancreatitis aguda; el número total de fallecidos por dolor abdominal agudo fue de 299 personas y para pancreatitis aguda de 194 personas [2].

El término "abdomen agudo" fue introducido a la literatura médica por John B Deaver, quien lo describe como: "cualquier problema intraabdominal agudo que requiere de tratamiento quirúrgico urgente." Mayo M., et al definen al abdomen agudo como una entidad compleja, mórbida, multisindrómica, de defensa aguda ante agresiones abdominales, que precisa un diagnóstico y tratamiento temprano. Puede estar causado por una agresión al compartimento peritoneal, con el dolor abdominal como síntoma predominante y desencadena una fisiopatología intraabdominal y sistémica, evolutivamente grave y rápida, que le da gran potencialidad de producir muerte [3].

En 1921, Sir Zachary Cope señaló la importancia de realizar una anamnesis y examen físico completo. Cope hizo especial hincapié en que no tomar una decisión dentro de las 8 o 10 horas puede implicar un serio peligro para la vida del paciente, y que un retraso de 2 horas equivale entre dos semanas y dos meses para alcanzar la recuperación del paciente [4].

Dentro de las patologías causantes de dolor abdominal agudo que requieren de un tratamiento inmediato encontramos: apendicitis aguda, aneurisma de la aorta abdominal, afectación del árbol biliar (colelitiasis, colecistitis y colangitis aguda), perforación apendicular o de una víscera hueca, obstrucción del intestino delgado o grueso con o sin estrangulamiento, isquemia intestinal, pancreatitis aguda y severa, la rotura de un embarazo ectópico, rotura del bazo, etc. [3-5].

La pancreatitis aguda es una de las causas de dolor abdominal y para su diagnóstico la amilasa y lipasa sérica son los principales biomarcadores utilizados [3]. La amilasa y la lipasa son enzimas digestivas liberadas por los acinos celulares del páncreas exócrino hacia el duodeno. Cualquier daño pancreático libera grandes cantidades de estas enzimas a circulación. La lipasa sérica es la prueba preferida por su mayor sensibili- dad, particularmente en la pancreatitis inducida por el alcohol y por hipertrigliceridemia [6]. Su elevación prolongada crea una ventana de diagnóstico más amplia que la amilasa. En la pancreatitis aguda, la amilasa puede aumentar rápidamente dentro de las 3-6 horas posteriores a la aparición de los síntomas y puede permanecer elevada durante hasta cinco días. La lipasa generalmente alcanza un máximo a las 24 horas, con concentraciones séricas que permanecen elevadas durante 8-14 días. Esto significa que es mucho más útil que la amilasa cuando la presentación clínica o las pruebas se han retrasado por más de 24 horas [7].

Sin embargo numerosas investigaciones demuestran valores aumentados de amilasa y lipasa por encima de tres veces el límite superior de referencia, en casos de insuficiencia renal aguda, fallo renal crónico, enfermedades gastroduodenales e intestinales, patologías neuroquirúrgicas, etc., lo que complica la toma de decisiones médicas al momento de realizar el diagnóstico e instauración del tratamiento [8].

La importancia de este estudio radica en evaluar la utilidad de la amilasa y lipasa sérica en el diagnóstico y control de tratamiento de pacientes con dolor abdominal agudo de origen pancreático y no pancreático, permitiendo al médico tomar una mejor decisión ante la presencia de valores elevados de amilasa y lipasa sérica y no se sospeche de una etiología pancreática.

\section{Parte experimental}

El estudio es de enfoque mixto, nivel descriptivo correlacional, de tipo retrospectivo. La muestra incluyó a 72 pacientes atendidos en Northospital durante el periodo octubre a diciembre del 2015 que presentaron valores elevados de amilasa y lipasa sérica. Las variables que se estudiaron fueron obtenidas de las historias clínicas del departamento de Archivo y Estadística del Hospital, las cuales son: edad, sexo, antecedentes patológicos y familiares, motivo de consulta, signos y síntomas, pruebas de laboratorio e imagen, diagnóstico y tratamiento. Los analitos fueron procesados en el equipo automatizado Ortho Clinical Vitros 250 Chemistry System de la compañía Johnson \& Johnson de acuerdo a las especificaciones para cada analito. Para el análisis bioestadístico se utilizó los programas IBM SPSS Statistics versión 24 y MedCalc® versión 13.3.

\section{Resultados y discusión}

De los 72 pacientes incluidos en el estudio, el 58,3\% $(n=42)$ corresponden al género masculino y el $41,7 \%$ $(n=30)$ corresponden al género femenino. La edad me- 
dia de los pacientes es de 60.67 años, el rango comprende entre 16 y 96 años, el valor de la mediana es de 64 años, la desviación estándar es de 19.74. En la tabla 1 se presenta la distribución de frecuencias y porcentajes de las patologías de origen pancreático y no pancreático.

Tabla 1. Patologías con valores elevados de amilasa y lipasa sérica

\begin{tabular}{ccc}
\hline Patologías & Frecuencia, (n) & Porcentaje, \% \\
\hline Patología Pancreática & 18 & 25 \\
Patología no Pancreática & 54 & 75 \\
\hline Total & 72 & 100,0 \\
\hline
\end{tabular}

Fuente: Historias clínicas. Departamento Archivo y Estadística Northospital

\subsection{Patologías diferentes a la pancreatitis aguda con aumentos de amilasa $y$ lipasasérica}

Se observó patologías diferentes a la pancreatitis aguda que presentan valores elevados de lipasa sérica (Tabla 2) como es el caso de: hemorragia subaracnoidea $(3,0 N)$, insuficiencia renal crónica $(3,3 \mathrm{~N})$, vesícula necrótica perforada $(2,9 \mathrm{~N})$, diverticulosis del colon $(2,7 \mathrm{~N})$, etc., siendo éstas enfermedades de importancia médica ya que sus elevaciones de tres veces el valor normal $(3 \mathrm{~N})$ podrían conducir a un diagnóstico incorrecto en los pacientes asintomáticos.

Tabla 2. Patologías con aumentos de amilasa y lipasa sérica

\begin{tabular}{|c|c|c|c|c|}
\hline Patologías & Frecuencia, (n) & Porcentaje, \% & Amilasa sérica $(U / l) \bar{X}$ & Lipasa Sérica (U/I) $\bar{X}$ \\
\hline Coledocolitiasis & 22 & 30,6 & $144,6(1,3 N)$ & $488,3(1,6 \mathrm{~N})$ \\
\hline Colelitiasis & 19 & 26,4 & $190,5(1,7 \mathrm{~N})$ & $383,4(1,3 \mathrm{~N})$ \\
\hline Colecistitis & 12 & 16,7 & $159,6(1,5 \mathrm{~N})$ & $273,8(0,9 \mathrm{~N})$ \\
\hline Colangitis & 8 & 11,1 & $115,8(1,7 N)$ & $458,8(1,5 \mathrm{~N})$ \\
\hline Hemorragia subaracnoidea & 3 & 4,2 & $175,3(1,6 \mathrm{~N})$ & $839,3(3,0 \mathrm{~N})$ \\
\hline Insuficiencia renal crónica & 2 & 2,8 & $129(1,2 N)$ & $1008(3,3 \mathrm{~N})$ \\
\hline Vesícula necrótica perforada & 1 & 1,4 & $174(1,6 \mathrm{~N})$ & $872(2,9 \mathrm{~N})$ \\
\hline Ampuloma & 1 & 1,4 & $418(3,8 \mathrm{~N})$ & $1269(4,3 N)$ \\
\hline Diverticulosis de colon & 1 & 1,4 & $242(2,3 N)$ & $801(2,7 \mathrm{~N})$ \\
\hline Pancreatitis crónica & 1 & 1,4 & $419(3,8 \mathrm{~N})$ & $1521(5,1 \mathrm{~N})$ \\
\hline Tumor maligno páncreas & 1 & 1,4 & $772(7,0 \mathrm{~N})$ & $8903(29,6 \mathrm{~N})$ \\
\hline Pancreatitis aguda grave & 1 & 1,4 & $4320(39,3 \mathrm{~N})$ & $12680(42 N)$ \\
\hline
\end{tabular}

$\mathbf{N}$ representa el valor del límite superior del intervalo normal, para la lipasa sérica corresponde a $300 \mathrm{U} / \mathrm{l}$ y para la amilasa sérica corresponde a $110 \mathrm{U} / \mathrm{I}$ valores de referencia del equipo automatizado Ortho Clinical Vitros 250 Chemistry System de la compañía Johnson \& Johnson. El número que antecede a $\mathrm{N}$ representa la relación entre la concentración de amilasa o lipasa sérica alcanzado en cada patología para el valor superior del intervalo normal de cada enzima pancreática.

Fuente: Historias clínicas. Departamento Archivo y Estadística Northospital. 


\subsection{Análisis comparativo entre los indica- dores de validez y seguridad diagnós- tica de la amilasa y lipasa sérica en el diagnóstico de pancreatitis aguda}

Se utilizó como indicadores de validez y seguridad diagnóstica a la sensibilidad, especificidad, valor predictivo positivo, valor predictivo negativo (Tabla 3) previo a la verificación del grado de correlación entre la amilasa y lipasa sérica frente a la ecografía abdominal utilizando el coeficiente de kappa de Cohen obteniéndose para la lipasa sérica vs la ecografía abdominal el valor de 0,815 $(0,80-1,0)$ indicando que existe una muy buena concordancia entre ambos resultados, y $0,312(0,20-0,40)$ para la amilasa sérica, indicando que existe una escasa concordancia en los resultados del análisis de esta enzima y la ecografía abdominal.

Tabla 3. Evaluación diagnóstica de biomarcadores de pancreatitis aguda

\begin{tabular}{ccccc}
\hline Biomarcadores & Sensibilidad & Especificidad & VPP & VPN \\
\hline Amilasa sérica & 0,64 & 0,75 & 0,88 & 0,43 \\
Lipasa sérica & 1,00 & 0,75 & 0,92 & 1,00 \\
\hline
\end{tabular}

VPP valor predictivo positivo, VPN valor predictivo negativo

Fuente: Historias clínicas. Departamento Archivo y Estadística Northospital.

\subsection{Establecimiento de puntos de corte me- diante Curvas Roc}

Al realizar el análisis de los biomarcadores de pancreatitis aguda mediante las curvas ROC se observa un área bajo la curva para la amilasa sérica de 0,727 (IC 95\%: 0,443 a 0,919) la cual es mayor de 0,50 con un valor $p>0,05$. El punto de corte para la amilasa sérica es de $>350 \mathrm{U} / /$ presentando una sensibilidad del 63,6\% (IC 95\%: 30,8-89,1), especi- ficidad 100\% (IC 95\%: 19,4-99,4), VPP 100\%, VPN $50 \%$ (Figura 1).

El área bajo la curva para la lipasa sérica es de 0,997 (IC 95\%: 0,745 a 0,100) la cual es mayor de 0,50 con un valor $p<0,05$ lo que nos indica que es estadísticamente significativo. El punto de corte para la lipasa sérica es de $>948 \mathrm{U} / /$ presentando una sensibilidad del 90,91\% (IC 95\%: 58,7-99,8), especificidad 100\% (IC 95\%: 39,8-100,0), VPP 100\%, VPN 80\% (Figura 2)
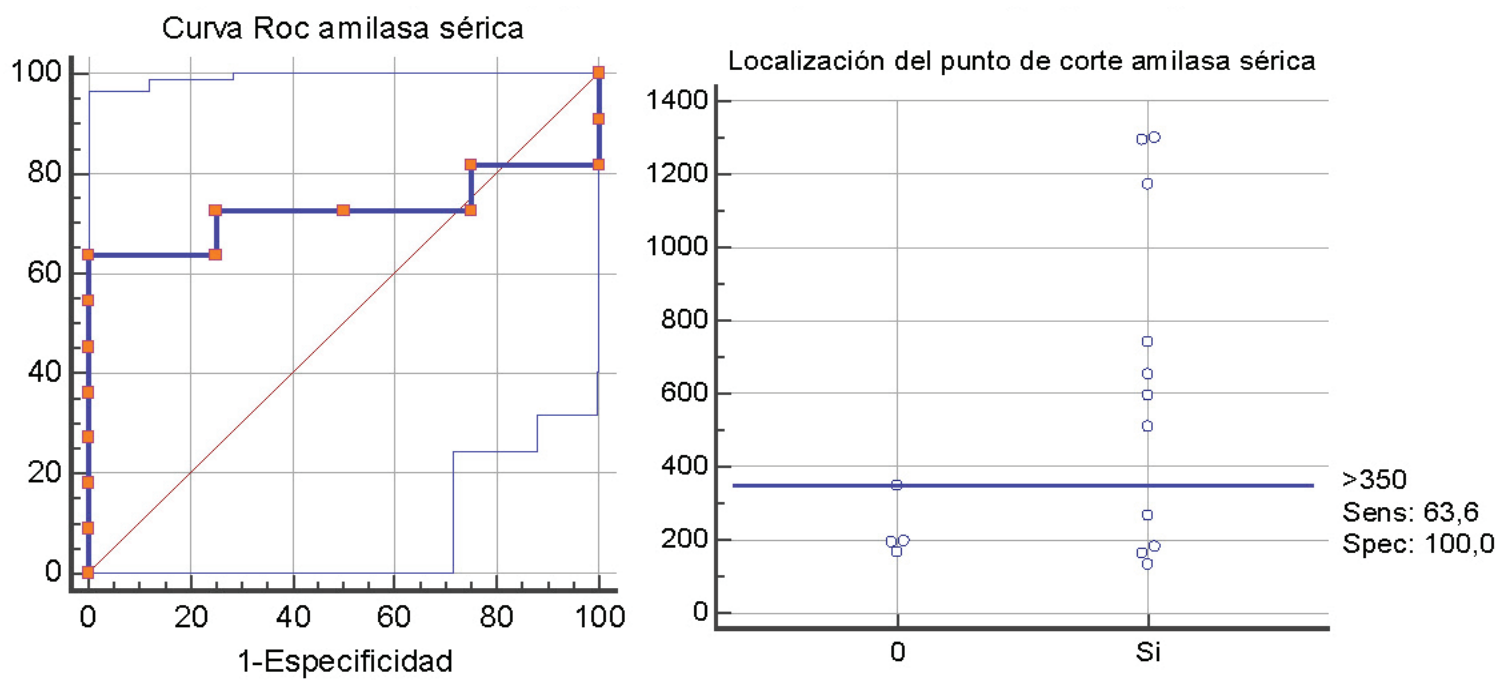

Figura 1. Curvas Roc amilasa sérica y ubicación del punto de corte

Fuente: Historias clínicas. Departamento Archivo y Estadística Northospital. 

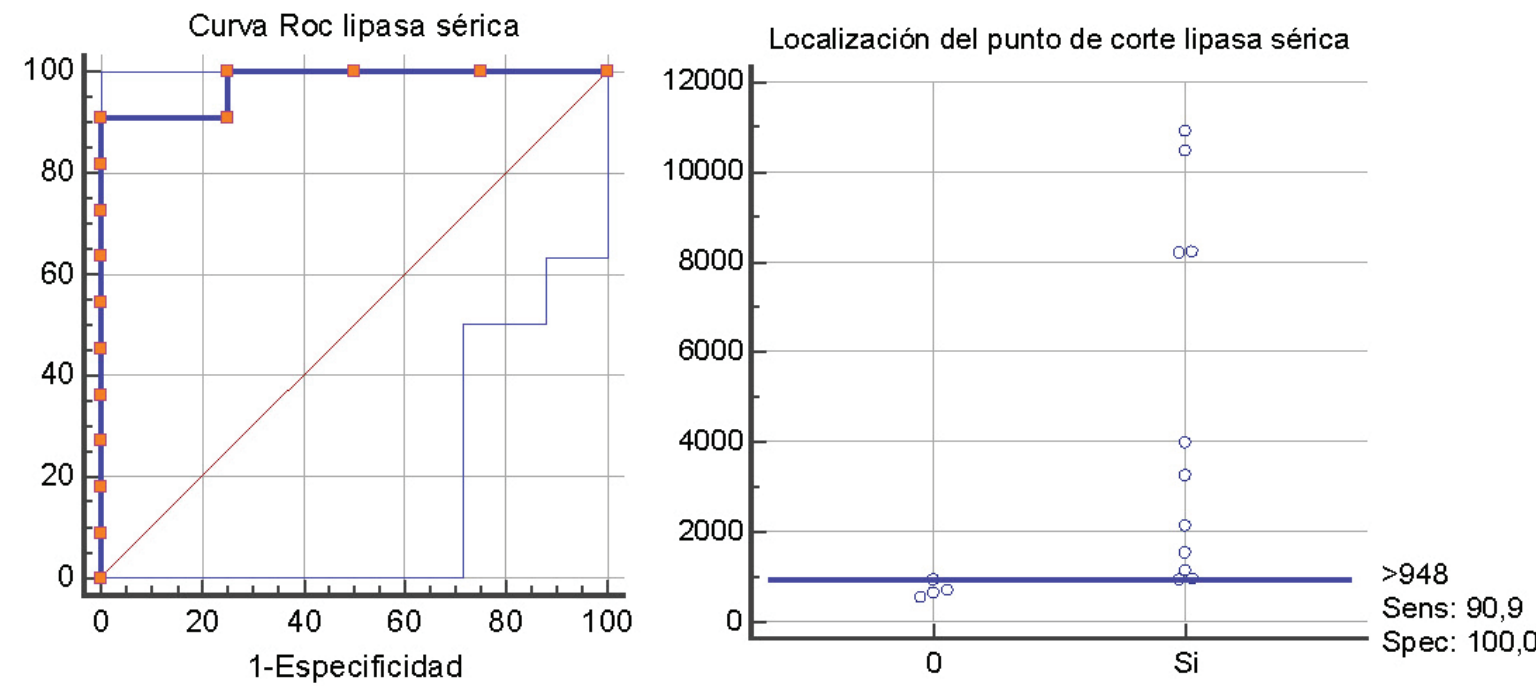

Figura 2. Curva Roc lipasa sérica y ubicación del punto de corte

Fuente: Historias clínicas. Departamento Archivo y Estadística Northospital.

\subsection{Otros biomarcadores para el diagnóstico de patologías no pancreáticas}

Con la finalidad de diferenciar las patologías no pancreáticas con afectación de la vesícula y vías biliares de la pancreatitis aguda se emplea la prueba estadística
Anova de un factor y prueba HSD Tukey para observar la elevación de las enzimas hepáticas, bilirrubina y leucocitos entre la coledocolitiasis, colelitiasis, colecistitis y colangitis. No se incluye el análisis de los valores de la aspartato aminotransferasa TGO/ASAT ya que no presentó elevaciones en estas enfermedades (Tabla 4).

Tabla 4. Análisis de la varianza con un factor

\begin{tabular}{|c|c|c|c|c|c|}
\hline Anova TGP/ALAT & $\begin{array}{l}\text { Suma de } \\
\text { cuadrados }\end{array}$ & gl & $\begin{array}{l}\text { Media } \\
\text { cuadrática }\end{array}$ & $\mathbf{F}$ & Sig. \\
\hline Entre grupos & 7865,441 & 3 & 2621,814 & 0,345 & 0,793 \\
\hline Dentro de grupos & 243069,309 & 57 & 7595,916 & & \\
\hline Total & 250934,750 & 60 & & & \\
\hline Anova Gamma GT & $\begin{array}{l}\text { Suma de } \\
\text { cuadrados }\end{array}$ & gl & $\begin{array}{l}\text { Media } \\
\text { cuadrática }\end{array}$ & $\mathbf{F}$ & Sig. \\
\hline Entre grupos & 83960,960 & 3 & 27986,987 & 1,465 & 0,243 \\
\hline Dentro de grupos & 611382,679 & 57 & 19105,709 & & \\
\hline Total & 695343,639 & 60 & & & \\
\hline $\begin{array}{c}\text { Anova Fosfatasa } \\
\text { alcalina }\end{array}$ & $\begin{array}{l}\text { Suma de } \\
\text { cuadrados }\end{array}$ & gl & $\begin{array}{l}\text { Media } \\
\text { cuadrática }\end{array}$ & $\mathbf{F}$ & Sig. \\
\hline Entre grupos & 82140,980 & 3 & 27380,327 & 1,319 & 0,285 \\
\hline Dentro de grupos & 664477,770 & 57 & 20764,930 & & \\
\hline Total & 746618,750 & 60 & & & \\
\hline
\end{tabular}

Fuente: Historias clínicas. Departamento Archivo y Estadística Northospital. 


\subsection{Evaluación del tratamiento de la pancrea- titis aguda mediante la utilización de la amilasa y lipasa}

Para observar la disminución en los valores de amilasa y lipasa sérica post tratamiento se utilizó la prueba t para muestras relacionadas previo la verificación de su normalidad empleando la herramienta estadística prueba de Kolmogorov-Smirnov, obteniéndose un valor $t$ de 3,894 para la amilasa sérica y 3,561 para la lipasa sérica, indicando que existe una disminución en los valores de sus concentraciones, siendo estas disminuciones estadísticamente significativas $\mathrm{p}<0,05$ (Tabla 4).

Tabla 5. Prueba t para muestras relacionadas

\begin{tabular}{ccc}
\hline Prueba $\boldsymbol{\dagger}$ & $\boldsymbol{\Delta}$ amilasa sérica U/I & $\boldsymbol{\Delta}$ lipasa sérica U/I \\
\hline Media amilasa antes & 561,07 & 3435,13 \\
Media amilasa después & 121,73 & 373,51 \\
Valor t & 3,984 & 3,561 \\
Grados de libertad & 14 & 14 \\
Significancia bilateral & 0,001 & 0,003 \\
\hline
\end{tabular}

${ }^{*} \mathrm{p}<0.05$ estadísticamente significativo

Fuente: Historias clínicas. Departamento Archivo y Estadística Northospital.

\subsection{Discusión}

Dentro de las principales causas de elevación de amilasa y lipasa sérica corresponden a una etiología pancreática el $25 \%$ ( $n=18$ casos), y a una etiología no pancreática el $75 \%(n=54)$.

El conocimiento de las enfermedades no pancreáticas que presentan elevación de amilasa y lipasa sérica tres veces el límite superior del valor normal, permite al médico tomar una mejor decisión ante la presencia de valores elevados de estas enzimas sin compromiso pancreático. Una de estas patologías es la hemorragia subaracnoideo de etiología aneurismática, la cual eleva en 3 veces el valor del límite superior del intervalo normal de la lipasa sérica observándose el mismo resultado en un estudio retrospectivo de 75 pacientes de una unidad de cuidados intensivos de neurocirugía en donde el $15 \%$ de los pacientes presentaron elevación de las enzimas pancreáticas por hemorragia subaracnoidea $(29 \%)$ y craneotomía $(24 \%)$. De estos pacientes ninguno presentaba evidencia clínica de pancreatitis aguda, concluyendo los autores que la causa de elevación de las enzimas se debe a una activación a través de vías centrales, tales como incremento del tono vagal, estimulación adrenérgica alterada, y/o la liberación de hormonas de activación tal como la colecistocinina (CCK)[9].
La insuficiencia renal crónica eleva en 3,3 veces el valor del límite superior del intervalo normal de la lipasa sérica y 1,2 veces el valor del límite superior del intervalo normal de la amilasa sérica. Resultados similares se observan en un estudio efectuado por Jiang et al., en el año 2002 quienes evaluaron las variaciones en las concentraciones de enzimas pancreáticas en pacientes con enfermedad renal crónica en comparación con controles sanos, encontrando que 28 pacientes $(60,7 \%)$ presentaban valores elevados de enzimas pancreáticas[10].

En nuestro estudio se tiene un caso clínico de un paciente con vesícula necrótica perforada que produce un aumento de 2,9 veces el valor del límite superior del intervalo normal de la lipasa sérica y 1,6 veces el valor del límite superior del intervalo normal de la amilasa. En la bibliografía en el año $2004 \mathrm{Kim}$ et al., reportan un caso clínico de un paciente mujer de 70 años de edad que presentó peritonitis causada por perforación de la vesícula con valores elevados de amilasa y lipasa sérica, pudiendo deberse a la estimulación de la secreción pancreática por acción de las sales biliares[ $[11]$.

Se encontró además un paciente con divertículos del colon y perforación del colon izquierdo que eleva en 2,7 veces el valor del límite superior del intervalo nor- 
mal de la lipasa y 2,3 veces el valor del límite superior del intervalo normal de la amilasa sérica. Al momento, en la bibliografía no se señalan casos de divertículos de colon o perforación del colon que presente elevación de enzimas pancreáticas. Sin embargo Miranda-Aquino et al., en el 2015 señalan que hasta en un $15 \%$ de los pacientes con pancreatitis grave o necrosis pancreática pueden observarse afectaciones en el colon, se cree que la fisiopatología es por extensión de las enzimas pancreáticas y la necrosis como causas principales [12].

En el caso del ampuloma, un tumor de la ampolla de Vater eleva en 4,3 veces el valor del límite superior del intervalo normal de la lipasa sérica y 3,8 veces el valor del límite superior del intervalo normal de la amilasa sérica. En una publicación de Vicente Lorenzo- Zúñiga et al., realizada en el 2007 señalan que se puede observar cuadros de pancreatitis aguda en pacientes con tumores de la ampolla de Vater secundarios a cuadros de obstrucción ductal a nivel pancreático [13].

Con la finalidad de realizar un mejor diagnóstico de pancreatitis aguda se evalúo a los biomarcadores amilasa y lipasa sérica mediante indicadores de validez, encontrándose que la lipasa sérica posee mayor sensibilidad $100 \%$, valor predictivo positivo $92 \%$, y guarda una buena concordancia con los resultados obtenidos de la ecografía abdominal (medida de acuerdo Kappa 0,815) en comparación con la amilasa sérica. La Sociedad Americana de Patología Clínica entre sus directrices y recomendaciones actuales al 2017, refiere que debe preferirse la determinación de lipasa a la amilasa total y pancreática para el diagnóstico inicial de pancreatitis aguda y que la evaluación no debe repetirse a lo largo del tiempo para controlar el pronóstico de la enfermedad. La repetición de la prueba debe considerarse solo cuando el paciente tiene signos y síntomas de inflamación pancreática o peripancreática persistente, obstrucción del conducto pancreático o desarrollo de un seudoquiste. En general, se desaconseja la determinación conjunta de amilasa y lipasa, porque aumenta los costos y solo mejora marginalmente la eficacia diagnóstica en comparación con cualquiera de los marcadores solo [8]. Akhtar et al., sustentan entre las ventajas de determinar únicamente lipasa sérica: su mayor sensibilidad, mayor especificidad, rápida elevación y al hecho de permanecer elevada por un periodo de tiempo mayor [14]. Igualmente la guía práctica para pancreatitis aguda de la Asociación Italiana para el estudio del páncreas en la pancreatitis aguda del 2010, recomienda el uso de la lipasa sérica sobre la amilasa sérica para el diagnóstico de pancreatitis aguda [15]. Sin embargo, se debe tomar en cuenta que a más de la pancreatitis, patologías como colecistitis, isquemia intestinal, insuficiencia renal y tumores malignos provocan la elevación sérica de lipasa [6].

De los marcadores bioquímicos para el diagnóstico de pancreatitis aguda mediante el empleo de Curva Roc la amilasa sérica con una concentración $>350 \mathrm{U} / \mathrm{I}$ presenta una sensibilidad del 63,6\% (IC 95\%: 30,8-89,1), especificidad 100\% (IC 95\%: 19,4-99,4), VPP 100\%, VPN 50\%. Treacy et al., en el 2001 reporta que la amilasa sérica determinada al segundo día de iniciado el cuadro clínico con una concentración de > $176 \mathrm{U} / \mathrm{l}$ presenta una sensibilidad del $45 \%$ y una especificidad del $97 \%$ [15]. Butler et al., en el 2012 señala que la amilasa sérica con una concentración $>290 \mathrm{U} / \mathrm{l}$ presenta una sensibilidad del $65 \%$ y una especificidad $90 \%$ resultados similares a esta investigación [17]. Otro estudio con resultados semejantes a los de esta investigación es la presentada por Gómez et al., en el 2012, que establecen en un estudio retrospectivo realizado en 151 pacientes con pancreatitis aguda que la sensibilidad de la lipasa es del $96.6 \%$ y su especificidad es del $99.4 \%$, en contraste con la sensibilidad de la amilasa del $78.6 \%$ y una especificidad del $99.1 \%$ [18].

Con respecto a la lipasa sérica empleando Curva Roc, una concentración $>948 \mathrm{U} / \mathrm{I}$ presenta una sensibilidad del 90,91\% (IC 95\%: 58,7-99,8), especificidad 100\% (IC 95\%: 39,8-100,0), VPP 100\%, VPN 80\%. Treacy et al., en el 2001 reportan que la lipasa sérica determinada al segundo día de iniciado el cuadro clínico con una concentración de $>208 \mathrm{U} / \mathrm{I}$ presenta una sensibilidad del $67 \%$ y especificidad del $97 \%$ [16]. Butler et al., en el 2012 señalan que la lipasa sérica con una concentración $>800 \mathrm{U} / /$ presenta una sensibilidad del $90 \%$ y una especificidad del $95 \%$ resultados muy similares a esta investigación [17].

Debido a la existencia de un mayor número de patologías que presentan valores elevados de amilasa y lipasa sérica de etiología no pancreática con afección de la vesícula y vías biliares, se realizó un ANOVA con un factor para enzimas hepáticas, bilirrubina total, leucocitos y neutrófilos, encontrándose que en pacientes con coledocolitiasis, colecistitis, colelitiasis y colangitis hay una elevación significativa de la alanina aminotransferasa TGP/ALAT, gamma glutamiltransferasa y-GT y fosfatasa alcalina. En la publicación de Dolores Velázquez et al., realizada en el 2010 concluyen que el uso de la gamma-glutamiltransferasa permite realizar un diagnóstico de coledocolitiasis [19]., de igual forma López R. $X$., en el 2012 al evaluar la gamma glutamiltransferasa como factor predictivo temprano en 124 pacientes atendidos en el servicio de cirugía general del Hospital Enrique Garcés de la ciudad de Quito concluye que la 
gamma glutamiltransferasa es útil para el diagnóstico de patología obstructiva de la vía biliar [20].

Para evaluar el tratamiento de la pancreatitis aguda mediante el empleo de la amilasa y lipasa sérica se utilizó la prueba t para muestras relacionadas previo a la verificación de su normalidad utilizando la prueba de Kolmogorov-Smirnov ( $p>0,05)$, encontrándose que al registrar los valores de estas enzimas a los tres días de iniciado el cuadro clínico dolor abdominal agudo y los tres días posteriores al tratamiento farmacológico - quirúrgico colangiopancreatografía retrógrada endoscópica (CPRE) y/o colecistectomía laparoscópica (Colelap) se observa un descenso significativo $p<0,05$ indicando eficacia del tratamiento y mejoría del estado del paciente.

En la literatura se señalan casos clínicos de pacientes con aumentos de amilasa y lipasa sérica atribuidos al uso de fármacos, con la finalidad de ver su relación se evalúo los antecedentes farmacológicos de los pacientes, encontrándose el caso de un paciente con fractura de fémur sin posible causa aparente de enfermedad que produzca una elevación de las enzimas pancreáticas, pudiendo atribuirse al uso del fármaco losartan. Badalov et al., concluyen que el diagnóstico de pancreatitis aguda inducida por medicamentos es difícil de establecer, lograron relacionar a 55 fármacos con el desarrollo de pancreatitis, dentro de los cuales se encuentra el losartan, un antagonista del receptor de angiotensina II [21].

\section{Conclusiones}

- Tanto la determinación de lipasa como la de amilasa sérica, presentan una especificidad del $100 \%$ para pancreatitis aguda comparados con la ecografía abdominal.

- Comparando con la ecografía abdominal, la lipasa sérica presentó una sensibilidad del $90.91 \%$, mayor que el $63,6 \%$ de sensibilidad para pancreatitis aguda que se determinó para la amilasa sérica.

- Las disminuciones de amilasa y lipasa sérica post tratamiento son estadísticamente significativas, por lo que estas dos enzimas son mar- cadores bioquímicos que indican eficacia del tratamiento y mejoría del estado del paciente.

- La amilasa y lipasa sérica constituyen biomarcadores de importancia para el diagnóstico y control del tratamiento en pacientes con pancreatitis aguda, sin embargo el $75 \%$ de los pacientes incluidos en este estudio presentaban concentraciones elevadas de amilasa y lipasa séricas sin evidencia de una etiología pancreática.

- Las afectaciones a la vesícula biliar y vías biliares son las patologías más comunes que elevan las enzimas pancreáticas.

- Si bien la lipasa sérica es altamente recomendada por las guías médicas como un mejor marcador para el diagnóstico de pancreatitis, las patologías no pancreáticas encontradas que presentan valores elevados de lipasa sérica mayor a tres veces su concentración normal incluyen a la hemorragia subaracnoidea, insuficiencia renal crónica, vesícula necrótica perforada, diverticulosis del colon, ampuloma.

- Elevaciones de amilasa y lipasa séricas no patológicas y no asociadas a dolor abdominal agudo, pueden atribuirse al uso de determinados fármacos, un paciente presentó hiperamilasemia e hiperlipasemia sin otra evidencia etiológica que la administración de losartan.

- En pacientes con coledocolitiasis, colecistitis, colelitiasis y colangitis hay una elevación significativa de la alanina aminotransferasa TGP/ALAT, gamma glutamiltransferasa $\mathrm{y}-\mathrm{GT}$ y fosfatasa alcalina.

- No se pudo establecer la utilidad diagnóstica y el control de tratamiento de la amilasa y lipasa sérica en patologías de etiología no pancreática debido a la falta de puntos de corte para estas enzimas pancreáticas y prueba Gold estándar para cada patología.

\section{Agradecimiento}

Los autores agradecen al Dr. Juan Pablo Aguayo Director Médico de Northospital por brindar las facilidades para llevar adelante el trabajo de investigación. 


\section{Referencias}

1. Fernández, J. A. (2009). Validez de las pruebas diagnósticas realizadas a pacientes con dolor abdominal agudo en un servicio de urgencias hospitalario. Revista Española de Enfermedades Digestivas, 610-618. Obtenido de http://scielo.isciii.es/scielo.php?pid=S1130$01082009000900003 \&$ script=sci_arttext\&tlng=es

2. INEC. (17 de agosto de 2016). Anuario de Estadísticas Hospitalarias: Egresos y Camas 2014. Obtenido de http://www.ecuadorencifras. gob.ec/

3. Mayo Ossorio, M., Pacheco García J., Vázquez Gallego J. (2016). Abdomen agudo. Medicine - Programa de Formación Médica Continuada Acreditado. 363-379. https://doi.org/10.1016/j.med.2016.03.012

4. Brunicardi, C. (2011). Principios De Cirugía Schwartz (Novena ed.). España: McGraw-Hill Interamericana de España S.L.

5. Cortes, D. (25 de abril de 2016). Dolor abdominal. Recuperado el 5 de Mayo de 2016, de http://www.igerontologico.com/salud/ digestivo-salud/dolor-abdominal-6398.htm

6. Basnayake C, Ratnam D. (2015). Blood tests for acute pancreatitis. Aust Prescr. 128-130. pmid:26648641

7. American Society for Clinical Pathology (2017). Do not test for amylase in cases of suspected pancreatitis. Instead, test for lipase. Choosing Wisely; 2017. www.choosingwisely. org/clinician-lists/american-society-clinical-pathologytesting-for-amylase/. Accessed December 12, 2017.

8. Muniraj, T. (2015). PANCREATITIS OR NOT?--Elevated lipase and amylase in ICU patients. Jornal of Critical Care, 1370-5. Obtenido de http://www.ncbi.nlm.nih.gov/pubmed/26411523

9. Hameed, A. M. (2015). Significant elevations of serum lipase not caused by pancreatitis: a systematic review. HPB (Oxford), $99-112$. Obtenido de http://www.ncbi.nlm.nih.gov/pubmed/24888393

10. Jiang, C.-F., Ng, K.-W., Tan, S.-W., Wu, C.-S., Chen, H.-C., Liang, C.-T., \& Chen, Y.-H. (2002). Serum level of amylase and lipase in various stages of chronic renal insufficiency. Zhonghua Yi Xue Za Zhi = Chinese Medical Journal; Free China Ed, 49-54.

11. Kim, H. J., Park, S. J., Lee, S. B., Lee, J. K., Jung, H. S., Choi, C. K., \& Paik, S. Y. (2004). A Case of Spontaneous Gallbladder Perforation. The Korean Journal of Internal Medicine, 128-131. doi:10.3904/kjim.2004.19.2.128

12. Miranda-Aquino, T., Pérez-Topete, S. E., Guajardo-Esparza, J. M., \& González-González, J. A. (2015). Necrosis del colon secundaria a pancreatitis aguda. Reporte de un caso y revisión literaria. Revista de Gastroenterología de México, 25-29. doi:http://dx.doi. org/10.1016/j.rgmx.2015.09.003

13. Lorenzo-Zúñiga, V., Moreno De Vega, V., Domènech, E., \& Boix, J. (2009). Diagnóstico y tratamiento de los tumores de la papila de Vater. Gastroenterología y Hepatología, 81-89. doi:10.1016/j.gastrohep.2008.02.004

14. Akhtar, A., Surode R., Agrawal D. (2017). Measuring both serum amylase and lipase for acute pancreatitis lowers quality and raise cost. Cleveland Clinic Journal of Medicine, 670-672.

15. Pezzilli, R., Zerbi, A., Di Carlo, V., Bassi, C., Delle Fave, G. F., \& Working Group of the Italian Association for the Study of the Pancreas on Acute Pancreatitis. (2010). Practical guidelines for acute pancreatitis. Pancreatology: Official Journal of the International Association of Pancreatology (IAP), 523-535. doi:10.1159/000314602

16. Treacy, J., Williams, A., Bais, R., Willson, K., Worthley, C., Reece, J., ... Thomas, D. (2001). Evaluation of amylase and lipase in the diagnosis of acute pancreatitis. ANZ Journal of Surgery, 577-582. doi:10.1046/j.1445-2197.2001.02220.x

17. Butler, J., \& Mackway-Jones, K. (2012). Serum amylase or lipase to diagnose pancreatitis in patients presenting with abdominal pain. Emergency Medice Jorunal (EMJ), 430-431. doi:10.1136/emj.19.5.430

18. Gomez D, Addison A, De Rosa A, Brooks A, Cameron IC. (2012). Retrospective study of patients with acute pancreatitis: is serum amylase still required? BMJ Open, 2. http://dx.doi.org/10.1136/bmjopen-2012-001471

19. Dolores V., Medina A., Jesús Vega A. (2010). Factores predictivos para el diagnóstico temprano. Cirujano General, $39-44$.

20. López, R. X. (2012). Utilidad de la gammaglutamil transpeptidasa como factor predictor temprano de coledocolitiasis. Universidad Central del Ecuador. Postgrado de Cirugía General, 1-98.

21. Badalov, N., Baradarian, R., Iswara, K., Li, J., Steinberg, W., \& Tenner, S. (2007). Drug-induced acute pancreatitis: an evidence-based review. Clinical Gastroenterology and Hepatology: The Official Clinical Practice Journal of the American Gastroenterological Asocciation, 248-672. 